
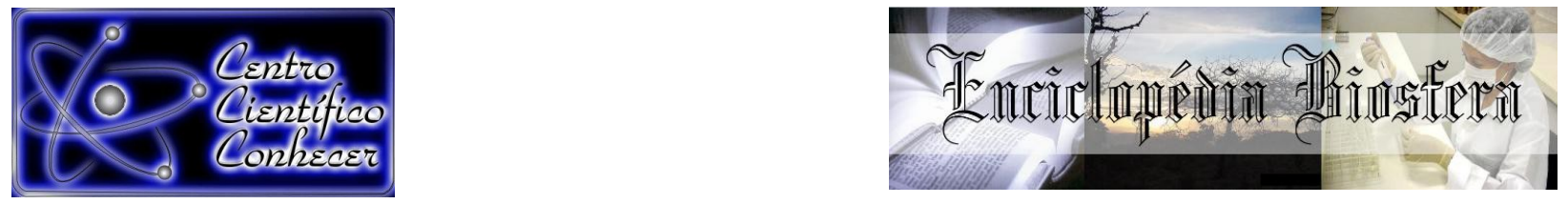

\title{
AVALIAÇÃO DA PRODUTIVIDADE DO PEPINO CONDUZIDO EM SISTEMAS DE TUTORAMENTO HORIZONTAL E VERTICAL COM E SEM CONSÓRCIO COM AMENDOIM EM RONDÔNIA
}

\author{
Jhonny Kelvin Dias Martins ${ }^{1}$, Silvana Ramlow Otto Teixeira da Luz ${ }^{1}$, Bruna Firmino \\ Enck $^{1}$, Geisibel Fernandes Keffer ${ }^{1}$, João Batista Dias Damaceno ${ }^{2}$ \\ ${ }^{1}$ Graduado (a) em agronomia, Fundação Universidade Federal de Rondônia - UNIR, \\ (jhonny.jkdm@gmail.com) Rolim de Moura- Brasil \\ ${ }^{2}$ Doutorando em Agronomia Tropical (Produção Vegetal), Fundação Universidade \\ Federal do Amazonas- UFAM, Manaus- Brasil
}

Recebido em: 06/04/2018 - Aprovado em: 10/06/2018 - Publicado em: 20/06/2018 DOI: 10.18677/EnciBio_2018A14

\begin{abstract}
RESUMO
A agricultura familiar está presente na maioria dos municípios do estado de Rondônia, o cultivo do pepino vem se destacando por participar cada vez mais da renda dos pequenos produtores, no entanto a falta de informações sobre o manejo adequado da cultura vem dificultando o aumento da produção. Portanto, objetivou-se com o presente trabalho avaliar o potencial produtivo do pepino (Cucumis sativus) submetido a dois sistemas de condução sendo um tutoramento horizontal e outro vertical, com e sem consórcio de amendoim (Arachis hypogaea). O experimento foi instalado no Campus experimental do curso de agronomia da Universidade Federal de Rondônia (UNIR), localizado no município de Rolim de Moura-RO. O delineamento experimental utilizado foi o em blocos casualizados, com quatro repetições e quatro tratamentos: 1-Tutoramento Vertical com Consórcio (TV-CC); 2Tutoramento Horizontal com Consórcio (TH-CC); 3-Tutoramento vertical sem consorcio (TV-SC); 4-Tutoramento Horizontal sem Consórcio (TH-SC). As variáveis analisadas foram diâmetro, comprimento e peso do fruto e produtividade média. Observou-se que quando o pepino em consórcio com amendoim e conduzido com tutoramento horizontal ( $\mathrm{TH}$ - $\mathrm{CC}$ ) obtém uma elevada produtividade, evidenciando que esse sistema é vantajoso para as condições climáticas do estado de Rondônia.
\end{abstract}

PALAVRAS-CHAVE: Agricultura familiar, Olericultura, Práticas culturais.

\section{EVALUATION OF THE PRODUCTIVITY OF CUCUMBER CONDUCTED IN HORIZONTAL AND VERTICAL TUNA SYSTEMS WITH AND WITHOUT CONSORTIUM WITH PEANUT IN RONDÔNIA}

\section{ABSTRACT}

Family farming is present in most of the municipalities of the state of Rondônia, cucumber cultivation has been highlighted by increasingly participating in the income of small producers, however the lack of information about the proper management of the crop has made it difficult to increase production. Therefore, the objective of this work was to evaluate the productive potential of cucumber (Cucumis sativus) submitted to two systems of conduction being horizontal and vertical, with and ENCICLOPÉDIA BIOSFERA, Centro Científico Conhecer - Goiânia, v.15 n.27; p.150 2018 
without peanut consortium (Arachis hypogaea). The experiment was installed in the experimental campus of the agronomy course of the Federal University of Rondônia (UNIR), located in the municipality of Rolim de Moura-RO. The experimental design was a randomized complete block design, with four replications and four treatments: 1-Vertical Tutoring with Consortium (TV-CC); 2-Horizontal Tutoring with Consortium (TH-CC); 3-Vertical tutoring without consortium (TV-SC); 4-Horizontal Tutoring without Consortium (TH-SC). The analyzed variables were fruit diameter, length and weight and average productivity. It was observed that when the cucumber in a consortium with peanuts and conducted with horizontal torturing ( $\mathrm{TH}-\mathrm{CC}$ ) obtained a high productivity, evidencing that this system is advantageous for the climatic conditions of the state of Rondônia

KEYWORDS: Family Farming, Olericultura, Cultural Practices

\section{INTRODUÇÃO}

O pepino (Cucumis sativus L.) pertence à família das cucurbitáceas, sendo originário da Ásia. Se adapta bem ao clima tropical, como no Brasil e é mais indicado o cultivo onde as temperaturas são mais elevadas e que não ocorra épocas de frio ou geadas (GOTO, 2014). Encontrando no estado de Rondônia condições favoráveis para o seu cultivo.

Segundo Amaro et al. (2014) o pepino está entre as 10 hortaliças de maior valor comercial no Brasil sendo que, dentre as cucurbitáceas, é uma das mais cultivadas em ambiente protegido, por permitir o cultivo intensivo e obtenção de altas produtividades. Possui várias cultivares e híbridos no mercado, com diferentes tamanhos, formas, coloração, sabor e características vegetativas (porte, hábito, ciclo, biologia reprodutiva) sendo classificados em cinco grupos distintos: aodai ou salada, caipira, japonês, holandês e industrial, também chamado de conserva (SEDIYAMA et al., 2014).

Embora possa ser conduzido de forma prostrada, o pepino tem o seu grande destaque quando conduzido em tutoramento, onde consegue elevada produtividade e ótima qualidade para consumo in natura. Outras vantagens do tutoramento: favorece o controle fitossanitário, facilita alguns tratos culturais, aumenta a longevidade da planta, alonga o período produtivo, favorece a colheita parcelada (FILGUEIRA, 2013).

De acordo com Barros Júnior et al. (2008) além do tutoramento têm-se os arranjos consorciados com outras culturas, o motivo principal pelo qual os produtores preferem o sistema de cultivo consorciado é justamente por obter maior produtividade quando comparado ao sistema de monocultura.

O consórcio tem inúmeras vantagens ao se analisar os benefícios para a preservação do solo, as leguminosas são as que vêm se destacando nesse tipo de manejo. O amendoim (Arachis Hypogaea L.) tem origem na América do Sul e é uma das principais leguminosas cultivadas no Brasil e no mundo, assim como o feijão e a soja (EMBRAPA, 2017).

É uma planta que tem condições ideais para ser consorciada com outras culturas, pois de acordo com Ferrari Neto et al. (2012) a cultura do amendoim é considerada como planta neutra, ou seja, não há efeito do fotoperíodo nas plantas, sendo que a luz não é um fator limitante para o desenvolvimento dessa cultura. Diante do exposto, o objetivo desse trabalho foi avaliar os efeitos de dois sistemas de tutoramento, submetidos ao sistema consorciado e não consorciado com amendoim, no rendimento produtivo do pepino. 


\section{MATERIAL E MÉTODOS}

O experimento foi realizado no Campus experimental do curso de agronomia da Universidade Federal de Rondônia (UNIR), localizado no município de Rolim de Moura-RO, na Amazônia ocidental. O campo experimental está localizado a $15 \mathrm{~km}$

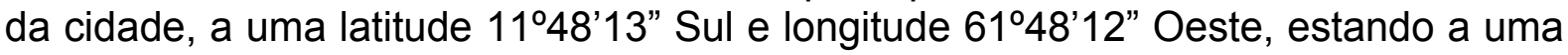
altitude de 290 metros. O clima é tropical quente e úmido com estações de seca bem definida (junho a setembro) com chuvas intensas nos meses de novembro a março (LAHORTA, 2014).

A precipitação média anual é de $2.250 \mathrm{~mm}$, umidade relativa do ar elevada no período chuvoso, em torno de $85 \%$. As temperaturas médias mínimas são de $24^{\circ} \mathrm{C}$ e as máximas de $32^{\circ} \mathrm{C}$ (SEDAM, 2016). O solo utilizado para o experimento foi um Latossolo Vermelho Amarelo Distrófico e de acordo com (segundo) a análise o solo apresenta as seguintes características químicas: $\mathrm{pH}$ em água $=5,6$; Matéria Orgânica $=19 \mathrm{~g} \cdot \mathrm{kg}^{-1} ; \mathrm{P}=3,7 \mathrm{mg} / \mathrm{dm}^{-3} ; \mathrm{K}=0,13 \mathrm{cmolc} \mathrm{dm}{ }^{-3} ; \mathrm{Ca}=1,3 \mathrm{cmolc} / \mathrm{dm}^{-3} ; \mathrm{Mg}$ : $0,3 \mathrm{cmolc} / \mathrm{dm}^{-3} ; \mathrm{H}+\mathrm{Al}=4,7 \mathrm{cmolc} / \mathrm{dm}^{-3} ; \mathrm{Al}=0,0 \mathrm{cmolc} \mathrm{dm}{ }^{-3} \mathrm{Argila}=215 \mathrm{~g} \cdot \mathrm{kg}^{-1}$.

O delineamento experimental utilizado foi em blocos casualizados, com quatro repetições e quatro tratamentos: 1-Tutoramento Vertical com Consórcio (TV-CC); 2Tutoramento Horizontal com Consórcio (TH-CC); 3-Tutoramento vertical sem consorcio (TV-SC); 4-Tutoramento Horizontal sem Consórcio (TH-SC). Cada parcela experimental foi composta por $4,30 \mathrm{~m} \times 2,25 \mathrm{~m}$.

As parcelas continham quatro linhas de pepino com espaçamento de $1,20 \mathrm{~m} \mathrm{x}$ $0,50 \mathrm{~m}$, sendo somente as duas linhas centrais consideradas úteis para efeito da coleta dos dados. As unidades consorciadas com amendoim tinham a mesma configuração das que não tinham consórcio. As linhas de amendoim foram espaçadas em $0,60 \mathrm{~m}$ entre linhas e em $0,30 \mathrm{~m}$ de cada lado das linhas onde estavam as plantas de pepino. $\mathrm{O}$ amendoim foi semeado no mesmo dia que $\mathrm{O}$ pepino.

A semente de pepino utilizada foi a híbrida do tipo caipira (Ginga), sendo semeadas três sementes por cova com adubações recomendadas por Trani et al. (2015). O crescimento das plantas foi acompanhado e quando necessário realizado tratos culturais, não sendo utilizadas podas ou capação. As linhas de tutoramento, tanto horizontal quanto vertical foram colocadas quando as plantas atingiram $0,30 \mathrm{~m}$ de altura. No caso do tutoramento horizontal, o tutoramento foi realizado semanalmente com linhas duplas espaças verticalmente $20 \mathrm{~cm}$ uma da outra com espaço entre elas de $7 \mathrm{~cm}$, colocando-se as plantas entre as fitas de forma que elas não se espalhassem.

As variáveis analisadas foram: Diâmetro do Fruto $(\mathrm{mm})$, Comprimento do Fruto $(\mathrm{cm})$, Peso do Fruto $(\mathrm{g})$ e Produtividade Média $\left(\mathrm{t} / \mathrm{ha}^{-1}\right)$. As avaliações iniciaram no pico de produção. Os frutos avaliados foram escolhidos de forma sistemática, para não comprometer os dados, sendo coletados 4 frutos por planta.

Os diâmetros dos frutos avaliados com o auxílio de um paquímetro manual e com auxílio de uma fita métrica foram medidos: o comprimento dos frutos, medindose de uma extremidade a outra e posteriormente pesando-se os frutos em uma balança digital. A produtividade quantificada com base nas colheitas feitas e com 0 peso total obtido de cada parcela, sendo feita a extrapolação para toneladas por hectare.

Os dados foram submetidos à análise de variância pelo teste $F(p \leq 0,05)$. Sendo os resultados significativos foi realizada a comparação de médias, utilizandose o teste de Tukey ao nível de $5 \%$ de probabilidade ASSISTAT 7.7 @ (SILVA; AZEVEDO, 2016). 


\section{RESULTADOS E DISCUSSÃO}

Houve efeito significativo entre os tratamentos, ao se analisar as vareáveis diâmetro do fruto $(p \leq 0,01)$ e peso do fruto $(p \leq 0,05)$. Já para o comprimento do fruto não houve efeito, o tratamento com consórcio conduzido com tutoramento horizontal (CC-TH) obteve um ganho no diâmetro do fruto de $20 \%$ comparado com esse mesmo manejo sem cobertura (SC-TH) (Tabela 1).

TABELA 1. Médias do diâmetro, comprimento e peso do pepino (Cucumis sativus) submetido ao Tutoramento Vertical com Consórcio (TV-CC); Tutoramento Horizontal com Consórcio (TH-CC); Tutoramento Vertical sem Consórcio (TV-SC); Tutoramento Horizontal sem Consórcio (TH$\mathrm{SC})$.

\begin{tabular}{lccc}
\hline Tratamentos & $\begin{array}{c}\text { Diâmetro do } \\
\text { Fruto } \\
(\mathrm{mm})\end{array}$ & $\begin{array}{c}\text { Comprimento do Fruto } \\
(\mathrm{Cm})\end{array}$ & $\begin{array}{c}\text { Peso do } \\
\text { Fruto } \\
(\mathrm{g})\end{array}$ \\
\hline TV-CC & & $10.16 \mathrm{a}$ & $200,95 \mathrm{~b}$ \\
TH-CC & $45,89 \mathrm{c}$ & $10.53 \mathrm{a}$ & $280,22 \mathrm{a}$ \\
TV-SC & $74,49 \mathrm{a}$ & $10.20 \mathrm{a}$ & $100,17 \mathrm{c}$ \\
TH-SC & $44,65 \mathrm{c}$ & $10.67 \mathrm{a}$ & $238,50 \mathrm{a}$ \\
\hline F & $59,35 \mathrm{~b}$ & & \\
\hline $\mathrm{CV}(\%)$ & $0.4850^{* *}$ & $1.9747^{\text {ns }}$ & $0.4514^{*}$ \\
\hline
\end{tabular}

ns= não significativo, ${ }^{* *} e^{*}$ significativo ao nível de $1 \%$ e $5 \%$, respectivamente, pelo teste $\mathrm{F}$. Médias seguidas pela mesma letra não difere entre si pelo teste de Tukey, ao nível de $5 \%$ de probabilidade.

Segundo Sediyama et al. (2014) o uso de culturas que promovem a cobertura do solo tem inúmeros benefícios destacando-se a conservação da umidade, a menor variação da temperatura, a proteção contra erosão, essas vantagens proporcionam consideravelmente o aumento da produtividade. $\mathrm{O}$ efeito supressor $\mathrm{e}$, ou, alelopático sobre várias plantas espontâneas, diminuição dos custos de instalação de uma cultura principal (REZENDE et al., 2005).

O comprimento do fruto não foi afetado pelos tratamentos, obtendo-se uma média geral de 10,39 cm (Tabela 1). Resende e Flori (2003) afirmam que pepinos com comprimentos de 4 a $12 \mathrm{~cm}$ possuem características ideias para $\mathrm{o}$ processamento industrial do fruto, cujo principal destino é o fruto em conserva. Os frutos tendem a atingir um peso maior em cultivos onde o tutoramento é horizontal, o que pode ser explicado por apresentar menor quantidade de frutos quando comparado com o tutoramento vertical, aumentando assim o seu comprimento e peso.

Matos et al. (2012) analisaram diferentes métodos de condução no tomateiro e constataram que o tutoramento vertical proporcionou os menores resultados, os autores ressaltam que a condução em tutoramento vertical proporciona menor proteção do fruto, assim afetando diretamente a sua produção e qualidade.

Verificou-se que as duas maiores médias em relação ao diâmetro do fruto foram obtidas utilizando o tutoramento horizontal, no entanto ao se fazer uso do consórcio juntamente com o tutoramento horizontal (CC-TH) obteve-se um aumento de $15 \mathrm{~mm}$ comparado com o manejo sem o consórcio com tutoramento horizontal (SC-TH). Os resultados estão relacionados com a ausência do consórcio com 
amendoim, o que favoreceu uma maior incidência de plantas daninhas, aumentando-se a competitividade à cobertura com amendoim, evidenciando uma competitividade direta por nutrientes com o plantio do pepino.

Ao analisar a produtividade observou-se que houve efeito significativo $(p \leq 0,01)$ quando aplicado o tratamento consorciado com tutoramento horizontal $\left(71,40\right.$ t/ha $\left.{ }^{-1}\right)$ tendo um aumento de $50 \%$ na produtividade em comparação com a condução sem consórcio submetida a tutoramento vertical que obteve a menor média de produtividade (Tabela 2).

TABELA 2. Produtividade Média do pepino (Cucumis sativus) submetido a Tutoramento Vertical com Consórcio (TV-CC); Tutoramento Horizontal com Consórcio (TH-CC); Tutoramento Vertical sem Consórcio (TV-SC); Tutoramento Horizontal sem Consórcio (TH-SC).

\begin{tabular}{cc}
\hline Tratamentos & Produtividade $\left(\mathrm{t}^{\left.\mathrm{h} \mathrm{h}^{-1}\right)}\right.$ \\
\hline CC - TV & $45,73 \mathrm{~b}$ \\
CC - TH & $71,40 \mathrm{a}$ \\
SC - TV & $35,49 \mathrm{c}$ \\
SC - TH & $47,15 \mathrm{~b}$ \\
\hline CV (\%) & 15,06 \\
F & $1,469^{\star \star}$ \\
\hline
\end{tabular}

ns= não significativo, ${ }^{* *} e^{*}$ significativo ao nível de $1 \%$ e $5 \%$, respectivamente, pelo teste $\mathrm{F}$. Médias seguidas pela mesma letra não difere entre si pelo teste de Tukey, ao nível de $5 \%$ de probabilidade

De acordo com Silva et al. (2014) o manejo da cultura quando é consorciado com alguma leguminosa ou apenas fazendo o uso de práticas que visam a cobertura do solo tende a aumentar a produtividade. A baixa produtividade em sistemas com tutoramento vertical está relacionado com a limitação do aproveitamento da luminosidade necessária para um bom desenvolvimento do fruto, um fator importante para a redução nesse tipo de condução é que esse sistema é mais suscetível a ocorrer a danificação das partes reprodutivas. A produtividade média de pepino em condições que a cultura é bem conduzida e manejada varia entre 40 e 50 t/ha ${ }^{-1}$ para esses grupos (GALVANI et al., 2000).

Uma desvantagem do tutoramento vertical é há necessidade constante de fixar as novas brotações, especialmente na época de maior produção, para manter a formação do sistema dossel. Já o tutoramento horizontal permite maior penetração da luz em sua parte inferior e sua distribuição mais uniforme sobre a superfície foliar. Portanto, há maior produção de frutos de melhor qualidade.

\section{CONCLUSÃO}

O pepino ao ser conduzido com tutoramento horizontal obtém um aumento no rendimento produtivo mostrando-se uma alternativa para as condições edafoclimáticas do estado de Rondônia. O plantio de pepino consorciado com amendoim proporciona um ganho significativo na produtividade. 


\section{REFERÊNCIAS}

AMARO, A. C. E.; MACEDO, A. C.; RAMOS, A. R. P.; GOTO, R.; ONO, E. O.; RODRIGUES, J.D. The use of grafting to improve the net photosynthesis of cucumber. Theoretical and Experimental Plant Physiology, v.26, n.3, p.241-249, 2014. <https://doi.org/10.1007/s40626-014-0023-1> doi: 10.1007/s40626-014-0023-1

BARROS JÚNIOR, A. P.; REZENDE, B. L. A.; CECÍLIO FILHO, A. B.; MARTINS, M. I. E. G.; PÔRTO, D. R. Q. Custo de produção e rentabilidade de alface crespa e americana em monocultura e quando consorciada com rúcula. Revista Caatinga, Mossoró, v.21, n.2 p.181- 192, 2008.

EMBRAPA-Empresa Brasileira de Pesquisa Agropecuária. Informação e tecnologia. Amendoim arvore do conhecimento, 2017. Disponível < http://www.agencia.cnptia.embrapa.br/gestor/agroenergia/arvore/CONT000fbl23vn00 2wx5eo0sawqe3ex35v9p.html . Acessado em 02 de março 2018.

FERRARI NETO, J.; DA COSTA, C. H. M.; CASTRO, G. S. A. Ecofisiologia do amendoim. Scientia Agraria Paranaensis, v. 11, n. 4, p.1-13, 2012. <http://dx.doi.org/10.1818/sap.v11i4.6033> doi: 10.1818/sap.v11i4.6033

FILGUEIRA, F. A. R. Novo manual de olericultura: Agrotecnologia moderna na Produção e comercialização de hortaliças. Viçosa: UFV, ed.3, p.421, 2013.

GALVANI, E.; ESCOBEDO J. F.; CUNHA A. R.; KLOSOWSKI, E. S. 2000. Estimativa do índice de área foliar e da produtividade de pepino em meio protegido Cultivos de inverno e de verão. Revista Brasileira de Engenharia Agrícola e Ambiental. $\quad v \quad 4: \quad$ p. $8-13,2000 . \quad<$ http://dx.doi.org/10.1590/S141543662000000100002> doi:10.1590/S1415-43662000000100002

GOTO, R. Programa brasileiro para a modernização da horticultura: normas de classificação do pepino. São Paulo: CQH/CEAGESP. 2014. Disponível em: $<$ http://www.ceagesp.gov.br/wp-content/uploads/2015/07/pepino.pdf>. Acesso em: 10 de janeiro /2018.

LAHORTA - Laboratório de Horticultura Tropical e Apicultura. KOPPEN-GEIGER, uptader world map of Köppen-Geiger climate classification. Hydrol. Earth System Science., 11, 1633-1644, 2014. Dísponível em: <www.lahorta.acagea.net>. Acesso em: 20. Jun. 2018.

MATOS, E. S.; SHIRAHIGE, F. H.; de MELO, P. C. Desempenho de híbridos de tomate de crescimento indeterminado em função de sistemas de condução de plantas. Horticultura brasileira, v. 30, n. 2, 2012. <http://dx.doi.org/10.1590/S010205362012000200010> doi: 10.1590/S0102-05362012000200010

RESENDE, M. G.; FLORI, J. E. Produtividade do Pepino para Processamento no Vale do São Francisco. Pesquisa Agropecuária Brasileira, Brasília, v. 38, n. 2, p. 251-255, 2003.

REZENDE, B. L. A.; COSTA, C. C.; CECÍLIO FILHO, A. B.; MARTINS, M. I. E. G. Custo de produção e rentabilidade da alface crespa, em ambiente protegido, em 
cultivo solteiro e consorciado com tomateiro, Jaboticabal, estado de São Paulo. Informações Econômicas, v. 35, n. 7, p. 42-50, jun. 2005.

SEDAM - Secretaria de Estado do Desenvolvimento Ambiental e COGEO Coordenadoria de Geociências. Boletim climatológico de Rondônia ano 2013.

Porto Velho, v.12, 2016. Disponível <em: http://sedamro.wix.com/sedam_ro_focosqueimada\#!qualidade-do-ar.> Acessado em 10 janeiro. 2018.

SEDIYAMA, M. A. N.; NASCIMENTO, J. L. M.; LOPES, I. P. C.; LIMA, P. C.; VIDIGAL, S. M. Tipos de poda em pepino dos grupos aodai, japonês e caipira. Horticultura Brasileira, Brasília, v. 32, n. 4, p. 491-496, 2014. < http://dx.doi.org/10.1590/S0102-053620140000400020> doi: 10.1590/S0102053620140000400020

SILVA, F. C. D.; CARVALHO, G. E.; PEREIRA, C. B.; TORRES, M. F. Avaliação da Produtividade do milho consorciado. Agrarian Academy, v.4, n.7; p. 2014 . < http://dx.doi.org/10.1677/Agrarian_Academy_2017a18>.

doi: 10.1677/Agrarian_Academy_2017a18

SILVA, F. de A. S. e.; AZEVEDO, C. A. V. de. The Assistat Software Version 7.7 and its in use the analysis of experimental data. African Journal de Agric.Res. v.11, n.39, p.3733-3740, 2016. <http://dx.doi.org/10.5897/AJAR2016.11522> doi: 10.5897/AJAR2016.11522

TRANI, P. E.; PASSOS, F. A.; DE ARAUJO, H. S. Calagem e adubação do pepino. Instituto Agronômico de campinas, Centro de Horticultura, 2015. Disponível < http://www.iac.sp.gov.br/imagem_informacoestecnologicas/98.pdf> acessado dia 12 de dezembro 2017. 